\title{
Proton Pump Inhibitors and Survival in Patients With Colorectal Cancer Receiving Fluoropyrimidine-Based Chemotherapy
}

\author{
Ganessan Kichenadasse, MBBS, FRACP ${ }^{1,2}$; John O. Miners, $\mathrm{PhD}^{1}$; Arduino A. Mangoni, MBBS, PhD, FRACP1;
} Christos S. Karapetis, MBBS, FRACP2; Ashley M. Hopkins, PhD'; and Michael J. Sorich, PhD ${ }^{1}$

\begin{abstract}
Background: Concomitant use of proton pump inhibitors (PPIs) may negatively affect the efficacy of anticancer drugs such as fluoropyrimidines in patients with colorectal cancer (CRC). The primary objective of this study was to assess whether there is an association between concomitant PPI use and survival outcomes in patients with CRC treated with a fluoropyrimidine-based chemotherapy. Patients and Methods: A secondary analysis of 6 randomized controlled clinical trials in patients with advanced CRC was conducted using individual patient data through data-sharing platforms. The outcome measures were progression-free survival and overall survival in PPI users and nonusers. Subgroup analysis included the type of chemotherapy, capecitabine versus 5-FU, line of therapy, and addition of a vascular endothelial growth factor receptor inhibitor. Overall pooled hazard ratios (HRs) with 95\% confidence intervals were calculated using a random effects model. Results: A total of 5,594 patients with advanced CRC across 6 trials and 11 trial arms were included; 902 patients were receiving a PPI at trial entry and initiation of chemotherapy. PPI use was significantly associated with worse overall survival (pooled HR, 1.20; $95 \% \mathrm{Cl}, 1.03-1.40 ; P=.02 ; P^{2}$ for heterogeneity $=69 \%$ ) and progression-free survival (overall pooled $\mathrm{HR}, 1.20 ; 95 \% \mathrm{Cl}, 1.05-1.37 ; P=.009 ; P^{2}=65 \%$ ) after adjusting for clinical covariates. Furthermore, the association between concomitant PPI use and survival outcomes was similar across most treatment subgroups. Conclusions: We speculate that alterations in the gut microbiome, altered immune milieu within the tumor, and interactions through transporters are potential mechanisms behind this association between PPI use and chemotherapy in patients with CRC, which warrant further study. Concomitant use of PPIs is associated with worse survival outcomes in patients with CRC treated with fluoropyrimidine-based chemotherapy. Clinicians should cautiously consider the concomitant use of PPls in such patients.
\end{abstract}

J Natl Compr Canc Netw 2021;19(9):1037-1044 doi: $10.6004 /$ jnccn.2020.7670

\footnotetext{
${ }^{1}$ Department of Clinical Pharmacology, College of Medicine and Public Health, and ${ }^{2}$ Department of Medical Oncology, Flinders Centre for Innovation in Cancer, Flinders Medical Centre/Flinders University, Bedford Park, South Australia, Australia.
}

\section{Background}

Proton pump inhibitors (PPIs) are one of the most commonly used drugs in patients with cancer, especially those with gastrointestinal malignancies. ${ }^{1}$ In observational studies in noncancer populations, the long-term use of PPIs was associated with several adverse outcomes, including increased all-cause mortality, cardiovascular and renal diseases, dementia, infections, fractures, hypomagnesemia, and cancers. ${ }^{2,3}$ In contrast, in preclinical in vitro studies, PPIs were initially reported to improve the efficacy of some anticancer agents through direct anticancer effects and altered acidity within the tumor microenvironment. ${ }^{4-7}$ More recently, studies have described the potential for PPIs to adversely affect cancer outcomes when administered concomitantly with oral anticancer drugs such as kinase inhibitors. ${ }^{8-11}$ An increased intragastric $\mathrm{pH}$ from PPI use, with consequent reduced absorption of kinase inhibitors, was considered the mechanism responsible for this interaction. ${ }^{9}$ Changes in the gastrointestinal microbiome are another mechanism through which PPIs may affect cancer outcomes and the metabolism of drugs. ${ }^{12}$

Observational studies and retrospective analyses of trial data have also shown, albeit not consistently, that PPIs may decrease the efficacy of capecitabine, an oral fluoropyrimidine. As a result, patients with colorectal cancer (CRC), breast cancer, and other malignancies treated with capecitabine might have an increased risk of cancer recurrence and/or shorter survival. ${ }^{13-18}$ However, pharmacokinetic and in vitro studies have failed to identify the mechanisms of the interaction between PPIs and capecitabine. ${ }^{19}$ Furthermore, it is unclear whether the potentially negative effects of PPIs might also involve intravenously administered cytotoxic agents.

We sought to address these issues by assessing the association between concomitant PPI use and survival

See JNCCN.org for supplemental online content. 
outcomes in patients with advanced CRC treated with a fluoropyrimidine-based chemotherapy regimens using data from 6 completed CRC clinical trials. Furthermore, we assessed whether this association differed between oral and systemically administered fluoropyrimidines and between other agents combined with the fluoropyrimidines.

\section{Patients and Methods}

\section{Study Population}

We performed a retrospective post hoc analysis using anonymized individual patient data from 6 clinical trials in patients with advanced CRC obtained through the data-sharing platforms Project Data Sphere ${ }^{20}$ and Clinical Study Data Request ${ }^{21}$ : the AVF2107g trial (ClinicalTrials.gov identifier: NCT00109070), ${ }^{22}$ the trial reported by Carrato et a ${ }^{23}$ (NCT00457691), the HORIZON III trial (NCT00384176), ${ }^{24}$ the VELOUR trial (NCT00561470), ${ }^{25}$ the N016966 trial (NCT00069095), ${ }^{26}$ and the RAISE trial (NCT01183780). ${ }^{27}$ Sponsors of the Carrato et al, HORIZON III, and VELOUR trials released data from their respective control arms only, whereas sponsors for the other 3 trials provided data for both control and intervention arms. The Southern Adelaide Clinical Health Research ethics committee exempted review for this analysis.

\section{Study Definitions}

A fluoropyrimidine-based regimen was defined as combination anticancer therapy including at least one of the fluoropyrimidines 5-FU or capecitabine. Fluoropyrimidine-based chemotherapy, given as either first-line or second-line therapy, included either irinotecan or oxaliplatin as part of multiagent combination therapy with fluoropyrimidines and leucovorin. Concomitant vascular endothelial growth factor receptor inhibitor (VEGFi) therapies administered were bevacizumab (BEV) or ramucirumab (RAM), depending on the trial. Concomitant PPIs used were esomeprazole, lansoprazole, omeprazole, pantoprazole, or rabeprazole. PPI use was defined as treatment with any PPI at the time of initiation of the respective trial intervention, for a minimum of 7 days.

\section{Data Collection and Outcomes}

Data extracted were age at trial enrollment, sex, race, tumor response, time to progression, and survival time. The outcome measures were progression-free survival (PFS) and overall survival (OS) among PPI users and nonusers. PFS and tumor response were assessed using RECIST or RECIST version 1.1. Best overall response was defined as combined complete and partial responses. Subgroup analysis for the association between PPI use and OS included the type of chemotherapy (oxaliplatin vs irinotecan), oral versus intravenous fluoropyrimidine administration, addition of VEGFi, and line of therapy.

\section{Statistical Analysis}

Analysis was conducted using cohorts with fluoropyrimidinetreated patients. Hazard ratios (HRs) with 95\% confidence intervals for the association between PPI use and survival outcomes were estimated individually for each trial arm using Cox proportional hazards regression. HRs were adjusted for age, sex, race, ECOG performance status (PS), and serum CEA and lactate dehydrogenase (LDH) levels. Patients for whom all these covariates were available were included for adjusted analysis. Estimates were then pooled across all trials and arms using random-effects meta-analysis methods. A fixed-effect meta-analysis model was applied as a sensitivity analysis. Trial and summary HRs were visually displayed using forest plots, and statistical heterogeneity was described using the $I^{2}$ statistic. Adjustment for other contemporary confounding factors that affect survival, such as RAS mutations, right-side versus left-side primary, and liver versus other sites of metastases, were performed only for the RAISE trial data and not for other trials because of either the lack of collected data or data not being released by the sponsors. Analysis was performed using R version 3.4.3 (R Foundation for Statistical Computing). A $P$ value $<.05$ was considered statistically significant.

\section{Results}

Data from 11 arms across 6 trials were available for analysis (supplemental eTable 1, available with this article at JNCCN.org). The N016966 trial had 4 arms, 2 trials had 2 arms, and the remainder had 1 arm. 5-FU with leucovorin and irinotecan (folinic acid/5-FU/irinotecan [FOLFIRI] or irinotecan/5-FU/leucovorin [IFL]) was the chemotherapy in all trials except N016966 and HORIZON III, in which a fluoropyrimidine was combined with oxaliplatin. Among the VEGFi therapies, BEV was combined with chemotherapy (BEV + IFL, BEV + folinic acid/5-FU/oxaliplatin [FOLFOX], or BEV + capecitabine/ oxaliplatin [CAPOX]) in the AVF2107g, HORIZON III, and N016966 trials, whereas ramucirumab (RAM) was combined with chemotherapy (RAM + FOLFIRI) in the RAISE trial. Data on sunitinib or aflibercept treatment arms were not available for analysis. Most of the included trials were first-line interventions, whereas VELOUR and RAISE involved second-line therapies. From a total of 5,633 patients initially identified as intention to treat, data from 5,594 were available for further analysis as 


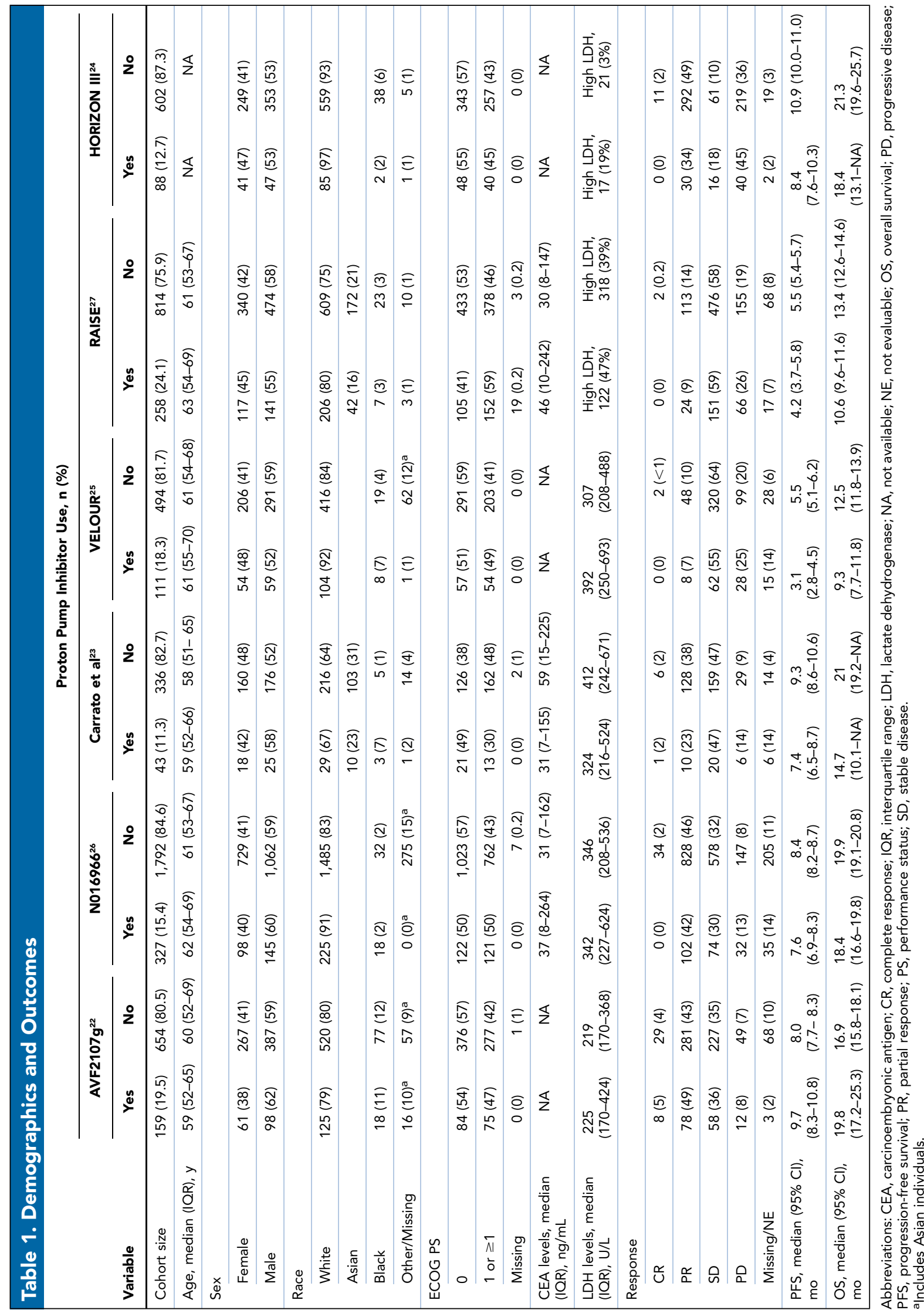


per-protocol treatment population. Their baseline characteristics are shown in Table 1. Most (58.8\%) were men, and the median age was 60 years.

\section{PPI Use}

A total of 902 patients were receiving a PPI at the start of the trial chemotherapy intervention. The proportion of PPI users ranged between $11.3 \%$ and $25.8 \%$ across the trial cohorts (supplemental eTable 2). PPI users had a similar median age and were more likely to be White compared with the non-PPI users. Omeprazole was the most frequently used PPI (39\%).

\section{Pooled Association of PPI Use and \\ Survival Outcomes}

Pooled analysis of the crude association between PPI use and survival outcomes indicated that PPI use was associated with statistically significant worse OS (random effects pooled HR, 1.23; 95\% CI, 1.07-1.43) (supplemental eFigure 1) and PFS (HR, 1.22; 95\% CI, 1.07-1.38) (supplemental eFigure 2). The association between PPI use and survival outcomes was then adjusted for age, sex, race, ECOG PS, and baseline CEA and LDH levels, when available, in 5,262 participants with complete data for the adjustment variables. Figure 1 shows the pooled estimates of adjusted HRs for OS between PPI users and nonusers during fluoropyrimidine-based chemotherapy. There was a statistically significant association between PPI use and worse OS outcomes with fluoropyrimidine-based chemotherapy (random-effects adjusted HR, 1.20; 95\% CI, $1.03-1.40 ; P=.02$ ) with substantial heterogeneity in effect size between studies $\left(I^{2}=69 \%\right)$. A sensitivity analysis using a fixed-effect model estimated a similar effect size (pooled HR, 1.20; 95\% CI, 1.10-1.30). Based on pooled analysis, a significant effect of PPI use on PFS with fluoropyrimidine-based chemotherapy (overall pooled HR, 1.20; 95\% CI, 1.05-1.37; $P=.009 ; I^{2}=65 \%$ ) was observed (Figure 2).

\section{Subgroup Analyses}

Subgroup analyses were performed to assess whether the adjusted association between concomitant PPI use and survival outcomes differed across key subgroups. Generally, estimates of association between PPI use and survival outcomes were relatively consistent across treatment subgroups for both OS and PFS (Figure 3). There was little evidence to support heterogeneity of effect size based on the chemotherapy agent (irinotecan vs oxaliplatin) combined with the fluoropyrimidine (supplemental eFigure 3A), the addition of a VEGFi to chemotherapy (supplemental eFigure 3B), or the line of therapy (supplemental eFigure 3C).

However, the comparison of subgroups treated with capecitabine versus intravenous 5-FU indicated a trend toward statistically significant heterogeneity of PPI effect size $\left(P_{\text {heterogeneity }}=.08\right)$ (supplemental eFigure 3D). This exploratory analysis highlights that, for patients treated with capecitabine, the concomitant use of PPI may not be associated with inferior OS and PFS and that further study is warranted with respect to effects of PPI use in these subgroups.

\section{PPI Use and Response Rates}

Complete response to the treatment intervention was uncommon $(<5 \%)$. Wide variations across trials were observed in partial response, stable disease, and progressive disease with ranges of $7 \%$ to $49 \%, 10 \%$ to $64 \%$, and $7 \%$ to $45 \%$, respectively (Table 1). Although the odds ratios (ORs) for objective response rates with concomitant PPI use were low in most cohorts, there was no statistically significant difference in response rates between PPI users and non-PPI users, except in the HORIZON III trial (OR, 0.51; 95\% CI, 0.32-0.82) (supplemental eTable 3). No significant effect of

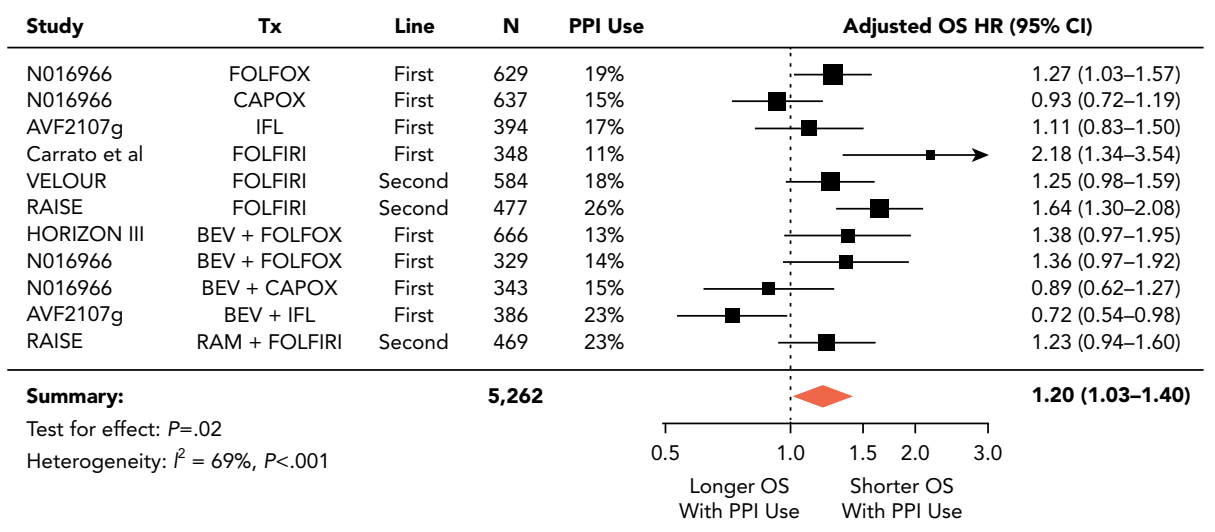

Figure 1. Pooled adjusted analysis of the association between PPI use and OS.

Abbreviations: BEV, bevacizumab; CAPOX, capecitabine/oxaliplatin; FOLFIRI, 5-FU/folinic acid/irinotecan; FOLFOX, 5-FU/folinic acid/oxaliplatin; HR, hazard ratio; $\mathrm{IFL}$, irinotecan/leucovorin/5-FU; OS, overall survival; PPI, proton pump inhibitor; RAM, ramucirumab; Tx, treatment. 


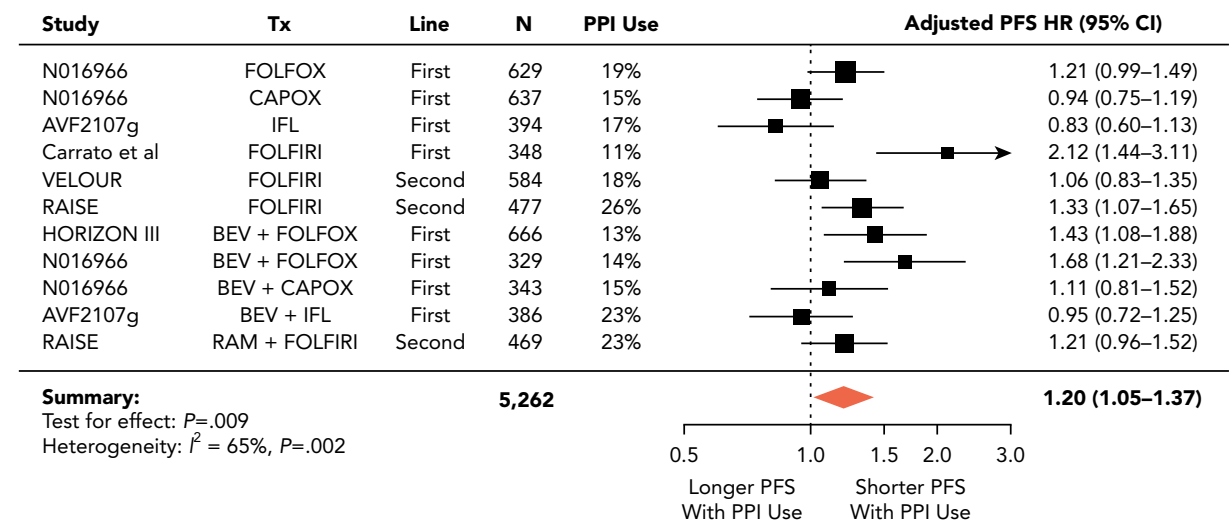

Figure 2. Pooled adjusted analysis of the association between concomitant PPI use and PFS.

Abbreviations: BEV, bevacizumab; CAPOX, capecitabine/oxaliplatin; FOLFIRI, 5-FU/folinic acid/irinotecan; FOLFOX, 5-FU/leucovorin/oxaliplatin; $\mathrm{HR}$, hazard ratio; IFL, irinotecan/leucovorin/5-FU; PFS, progression-free survival; PPI, proton pump inhibitor; RAM, ramucirumab; Tx, treatment.

concomitant use of PPI on adjusted overall response rates was observed (OR, 0.83; 95\% CI, 0.66-1.05; $P=.05 ; P^{2}=45 \%$ ) (supplemental eFigure 4).

\section{Additional Exploratory Analyses}

To explore whether the association arose from acid suppression or was specifically related to the use of a PPI, the effect of concomitant use of histamine H2-receptor antagonists (H2RAs) with fluoropyrimidine-based chemotherapy was evaluated in the same cohort of patients. A total of 362 patients (6.8\%) who received a concomitant H2RA were identified. There was no significant association between concomitant H2RA use and OS (adjusted HR, 0.98; 95\% CI, 0.86-1.12), PFS (adjusted HR, 0.97; 95\% CI, 0.85-1.11), and response rates (adjusted OR, 1.18; 95\% CI, 0.91-1.52) (supplemental eFigures 5, 6, and 7; supplemental eTable 4).
Additional contemporary prognostic factors, such as right-side versus left-side primary, KRAS mutation, and liver metastases, were available only for the RAISE trial. Hence, an exploratory analysis was performed by adding these variables to the other confounding factors just in these arms. After adjusting for multiple factors, the concomitant use of PPI had significantly worse OS and PFS, but not in the FOLFIRI + RAM arm (supplemental eTable 5).

\section{Discussion}

Results of this pooled analysis of 6 clinical trials in patients with advanced CRC receiving fluoropyrimidinebased combination chemotherapy indicate that concomitant PPI use is associated with significantly worse survival outcomes. This association was not seen with concomitant use of other acid-suppressing agents, such as H2RAs, indicating some degree of specificity with PPI use.

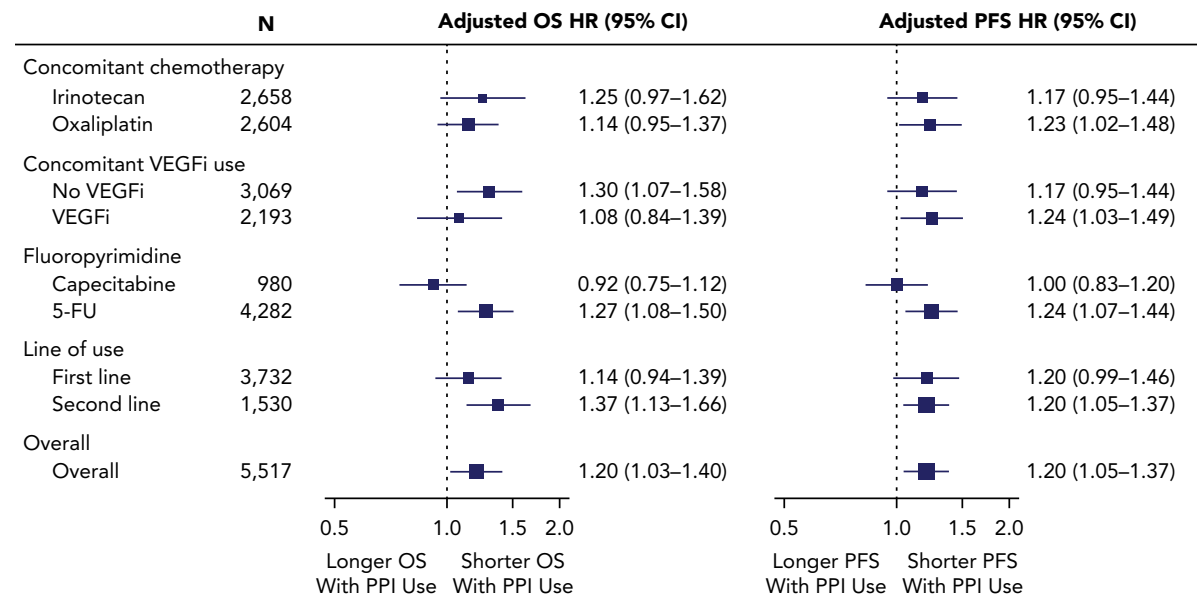

Figure 3. Association between concomitant PPI use and survival outcomes, by subgroup.

Abbreviations: HR, hazard ratio; OS, overall survival; PFS, progression-free survival; PPI, proton pump inhibitor; VEGFi, vascular endothelial growth factor receptor inhibitor. 
Substantial controversy remains regarding the potential negative effect of concomitant PPI use on cancer outcomes in patients undergoing fluoropyrimidine-based (especially capecitabine-based) chemotherapy. ${ }^{13,18,19,28,29} \mathrm{~A}$ retrospective series of 671 patients with CRC (474 receiving concomitant $\mathrm{PPI}$ ) reported improved survival in the FOLFOX-treated cohort but not in the CAPOX-treated cohort. ${ }^{29}$ However, using data from a prospective trial in patients with gastroesophageal cancers, Chu et al ${ }^{14}$ reported worse outcomes when PPIs were used concomitantly with CAPOX. Worse outcomes when PPIs were administered with capecitabine were also reported by other authors. ${ }^{16,17}$ In contrast, subgroup analyses in the present study did not find a significant negative association with survival (crude or adjusted) for concomitant PPI use across 980 patients treated with CAPOX (with and without BEV). However, there was a negative association with survival in the remaining patients treated with a range of 5-FU-based therapies. Our study did not include a cohort with monotherapy interventions with fluoropyrimidines, which prevents us from drawing any conclusions on the effect of PPIs on monotherapy. Moreover, the conflicting results between studies regarding an interaction between PPI use and add-on chemotherapy drugs indicate that the interaction is complex and may be context-specific. Prospective studies with complete data on PPI use, including duration of PPI use and treatment adherence, will help improve understanding of the association between PPI use of treatment outcomes. Similarly, the association between concomitant PPI use on the efficacy of other CRC drugs, such as anti-epidermal growth factor receptor (EGFR) inhibitors and trifluridine/tipiracil, warrants further evaluation.

Previous studies have identified that PPIs may have direct anticancer effects and also improve chemosensitivity of cancer cells by increasing extracellular pH. ${ }^{5,6,29}$ However, the concentration required to induce CRC cell death may not be reached in vivo. ${ }^{30} \mathrm{On}$ the contrary, as we have observed in this study, concomitant PPI use may be associated with negative effects on survival across the spectrum of combination chemotherapy with 5-FU. Although the lack of association observed with oral capecitabine (CAPOX or CAPOX + BEV) is intriguing, this association was based on data from a single study only (N016966), and the result of the test for heterogeneity did not reach statistical significance.

The mechanistic basis for the negative effect of concomitant PPI use is unclear. We speculate that PPIs may inhibit uptake transporters in tumor cells as one possible mechanism. It is well established that the chemotherapy drugs evaluated in this study are substrates for various uptake transporters, some of which are expressed in CRC cells. PPIs, especially omeprazole, have been reported to inhibit several transporters at therapeutic concentrations. For example, PPIs inhibit oxaliplatin uptake transporters such as organic anion transporters (OATs 1 and 3 ) and copper transporters, ${ }^{31}$ whereas irinotecan and its active form, $\mathrm{SN}-38$, inhibit transporters such as organic aniontransporting polypeptides (OATP1A/1B). ${ }^{32}$ However, PPIs may increase the expression of human equilibrative nucleoside transporter 1 (hENT1), potentially associated with poor response to fluoropyrimidines. ${ }^{33,34}$ It is also unclear whether there is a differential effect of PPIs on the intracellular uptake of oral versus intravenous fluoropyrimidines. In addition, the $\mathrm{pH}$ dependent uptake of chemotherapy drugs is well recognized ${ }^{35}$ It is possible that PPIs reduce the intratumoral concentration of cytotoxic drugs through the inhibition of uptake transporters and altered $\mathrm{pH}$ in the tumor microenvironment. This hypothesis requires further testing in preclinical studies.

Although chronic PPI use is associated with increased all-cause mortality in the general population, ${ }^{3}$ our study shows that both PFS and OS are negatively affected by concomitant PPI use with chemotherapy in patients with CRC. The negative association between PFS and PPI use during FOLFIRI chemotherapy, especially in the second-line setting, but not with IFL, indicates a possible interaction between irinotecan scheduling and antitumor response. A previous drug-drug interaction trial with short-term omeprazole and single-agent irinotecan did not show any significant changes in pharmacokinetic parameters and toxicities of irinotecan. ${ }^{36}$ Hence, it is likely that other mechanisms, such as alteration in gut microbiome, changes in tumor microenvironment and immune milieu by the PPIs, and their subsequent effects on irinotecan pharmacokinetics and pharmacodynamics, may play a role. ${ }^{4,737}$ Furthermore, the doses of 5-FU used in the IFL regimen versus FOLFIRI were different: a $500-\mathrm{mg} / \mathrm{m}^{2}$ bolus dose weekly for 4 out of 6 weeks was used in the IFL regimen, whereas both a bolus dose and an infusional dose of 5-FU were administered in the FOLFIRI regimen. The negative association was seen in the regimen with the infusion but not with the bolus schedule. The potential interaction between the dose and schedule of 5-FU and concomitant PPI should also be explored in future studies. Moreover, PPI-related microbiome changes can alter cancer outcomes through immunosuppression, increased drug metabolism, altered autophagy, or immunosuppression, thereby increasing resistance to 5 -FU and oxaliplatin. ${ }^{38-40}$ Further in vitro and in vivo studies will be required to confirm these findings.

This study assessed the effect of PPI use in patients with CRC undergoing fluoropyrimidine-based chemotherapy. 
It is unclear whether the negative effects of PPI use occur in other cancers and/or with other drugs, such as immunotherapies, and other targeted agents, such as EGFR inhibitors. Moreover, the use of PPIs may reflect the presence of other coexisting confounders, such as symptomatic advanced cancer with liver metastases, that may increase the need for PPIs. Future studies should consider addressing these gaps by exploring PPI use in early-stage CRC, non-CRC types, and other treatment settings.

Our study has significant strengths, including a relatively large cohort size, comprehensive prospective clinical trial data from individual patients, analysis using individual participant data, and pooled data from trials that included a variety of treatment regimens. However, it also has several limitations, including lack of analysis of the effect of comorbidities on OS and the impact of chemotherapy dose modifications; lack of information on the duration of PPI use before the start of the trial, dose/adherence to PPIs during the trial, initiation of PPIs after chemotherapy initiation, and the influence of other acid-suppressing drugs; lack of access to data from other trials with anti-EGFR inhibitors; and technical issues with data merging from various sharing platforms. None of the included trials had no-chemotherapy or nofluoropyrimidine arms for evaluation, thus precluding direct comparison and exploration of the prognostic relationship of concomitant PPI use. Further studies including an analysis of PPI use and survival outcomes in patients with metastatic CRC who are not receiving fluoropyrimidine-based chemotherapy are warranted. Such relationships could also be explored in other cancer types. Furthermore, most patients included in the trials were White individuals from the United States and Europe. Previous studies have reported regional differences in the safety of fluoropyrimidines, with East Asian individuals having the lowest incidence of adverse effects when compared with the US population, highlighting the need to evaluate the effects of concomitant PPI use among patients with different racial backgrounds and countries of origin. ${ }^{41}$

Although we adjusted for 6 clinically significant covariates in the calculation of pooled estimates, other contemporary prognostic factors such as right-sided versus left-sided location of the primary and molecular biomarkers such as RAS mutations and microsatellite instability were not uniformly available for inclusion. Moreover, we did not evaluate the association between PPI use and adverse effects during fluoropyrimidinebased chemotherapy or the actual cause of death to assess competing risk-based outcomes. Although future studies should consider these issues, accessing data derived from real-world use of PPIs during chemotherapy treatment may also provide further insights into this association.

\section{Conclusions}

Using data from 6 clinical trials including $>5,000$ patients with advanced CRC treated with fluoropyrimidine-based combination chemotherapy, we showed that concomitant use of PPIs was associated with worse OS and PFS. This association was significant after adjusting for age, sex, race, ECOG PS, and baseline CEA and LDH levels. The effect size of the association between PPI use and survival was similar across treatment subgroups, with the possible exception of capecitabine-based therapies, which require further evaluation. Pending identification of the mechanisms involved in this interaction and further confirmation in future studies, clinicians should cautiously consider the concomitant use of PPIs in patients with advanced CRC treated with fluoropyrimidinebased combination chemotherapy.

Submitted June 7, 2020; final revision received September 20, 2020; accepted for publication October 12, 2020

Published online May 5, 2021.

Author contributions: Study concept and design: Kichenadasse, Hopkins, Sorich. Data acquisition: Hopkins, Sorich. Data analysis and interpretation: All authors. Statistical analysis: Kichenadasse. Funding acquisition: Hopkins, Sorich.Technical/Material support: Miners, Mangoni, Hopkins. Supervision: Miners, Mangoni, Hopkins, Sorich. Manuscript preparation: All authors. Critical revision for intellectual content: All authors.

Disclosures: Dr. Karapetis has reported serving on the advisory board of Merck Serono, MSD, Bristol Myers Squibb, Roche, and AstraZeneca. Dr. Sorich has reported receiving grant/research support from Pfizer. The remaining authors have reported that they have not received any financial consideration from any person or organization to support the preparation, analysis, results, or discussion of this article.

Funding: This work was supported by funding from Cancer Council South Australia (1159924 and 1127220 to M.J. Sorich) and from the National Breast Cancer Foundation (PF-17-007 to A.M. Hopkins).

Correspondence: Ganessan Kichenadasse, MBBS, FRACP, Department of Medical Oncology, Flinders Centre for Innovation in Cancer, Flinders Medical Centre/Flinders University, Bedford Park, South Australia 5042, Australia. Email: ganessan.kichenadasse@flinders.edu.au

\section{References}

1. Smelick GS, Heffron TP, Chu L, et al. Prevalence of acid-reducing agents (ARA) in cancer populations and ARA drug-drug interaction potential for molecular targeted agents in clinical development. Mol Pharm 2013;10: 4055-4062.

2. Schoenfeld AJ, Grady D. Adverse effects associated with proton pump inhibitors. JAMA Intern Med 2016;176:172-174.

3. Xie $Y$, Bowe B, Yan $Y$, et al. Estimates of all cause mortality and cause specific mortality associated with proton pump inhibitors among US veterans: cohort study. BMJ 2019;365:11580.

4. Bellone $\mathrm{M}$, Calcinotto $\mathrm{A}$, Filipazzi $\mathrm{P}$, et al. The acidity of the tumor microenvironment is a mechanism of immune escape that can be overcome by proton pump inhibitors. Oncolmmunology 2013;2:e22058.

5. Ikemura K, Hiramatsu S, Okuda M. Drug repositioning of proton pump inhibitors for enhanced efficacy and safety of cancer chemotherapy. Front Pharmacol 2017;8:911.

6. Lugini L, Federici $C$, Borghi $M$, et al. Proton pump inhibitors while belonging to the same family of generic drugs show different antitumor effect. J Enzyme Inhib Med Chem 2016;31:538-545. 
7. Pilon-Thomas S, Kodumudi KN, El-Kenawi AE, et al. Neutralization of tumor acidity improves antitumor responses to immunotherapy. Cancer Res 2016;76:1381-1390.

8. Sharma M, Holmes HM, Mehta HB, et al. The concomitant use of tyrosine kinase inhibitors and proton pump inhibitors: prevalence, predictors, and impact on survival and discontinuation of therapy in older adults with cancer. Cancer 2019;125:1155-1162.

9. van Leeuwen RWF, Jansman FGA, Hunfeld NG, et al. Tyrosine kinase inhibitors and proton pump inhibitors: an evaluation of treatment options. Clin Pharmacokinet 2017;56:683-688.

10. van Leeuwen RW, van Gelder T, Mathijssen RH, et al. Drug-drug interactions with tyrosine-kinase inhibitors: a clinical perspective. Lancet Oncol 2014; 15:e315-326.

11. Hussaarts KGAM, Veerman GDM, Jansman FGA, et al. Clinically relevant drug interactions with multikinase inhibitors: a review. Ther Adv Med Oncol 2019:11:1758835918818347.

12. Wedemeyer RS, Blume $\mathrm{H}$. Pharmacokinetic drug interaction profiles of proton pump inhibitors: an update. Drug Saf 2014;37:201-211.

13. Altundag K. Coadministration of proton pump inhibitors may decrease the efficacy of capecitabine in metastatic breast cancer patients. J BUON 2017;22:559-560.

14. Chu MP, Hecht JR, Slamon D, et al. Association of proton pump inhibitors and capecitabine efficacy in advanced gastroesophageal cancer: secondary analysis of the TRIO-013/LOGiC randomized clinical trial. JAMA Oncol 2017;3:767-773

15. Graham C, Orr C, Bricks CS, et al. A retrospective analysis of the role of proton pump inhibitors in colorectal cancer disease survival. Curr Oncol 2016;23:e583-588.

16. Rhinehart HE, Phillips MA, Wade N, et al. Evaluation of the clinical impact of concomitant acid suppression therapy in colorectal cancer patients treated with capecitabine monotherapy. J Oncol Pharm Pract 2019;25: 1839-1845

17. Sun J, llich Al, Kim CA, et al. Concomitant administration of proton pump inhibitors and capecitabine is associated with increased recurrence risk in early stage colorectal cancer patients. Clin Colorectal Cancer 2016;15: 257-263.

18. Wong GG, Ha V, Chu MP, et al. Effects of proton pump inhibitors on FOLFOX and CapeOx regimens in colorectal cancer. Clin Colorectal Cancer 2019;18:72-79.

19. Cheng $\mathrm{V}$, Lemos $\mathrm{M}$, Hunter $\mathrm{N}$, et al. Concomitant use of capecitabine and proton pump inhibitors - is it safe? J Oncol Pharm Pract 2019;25:1705-1711.

20. Project Data Sphere. Project Data Sphere's Data Sharing Platform. Accessed December 20, 2019. Available at: https://www.projectdatasphere.org/projectdatasphere/html/home

21. ClinicalStudyDataRequest.com. Accessed December 20, 2019. Available at: https://www.clinicalstudydatarequest.com/Default.aspx

22. Hurwitz H, Fehrenbacher L, Novotny W, et al. Bevacizumab plus irinotecan, fluorouracil, and leucovorin for metastatic colorectal cancer. N Engl J Med 2004;350:2335-2342.

23. Carrato A, Swieboda-Sadlej A, Staszewska-Skurczynska M, et al. Fluorouracil, leucovorin, and irinotecan plus either sunitinib or placebo in metastatic colorectal cancer: a randomized, phase III trial. J Clin Oncol 2013;31:1341-1347.

24. Schmoll HJ, Cunningham D, Sobrero A, et al. Cediranib with mFOLFOX6 versus bevacizumab with mFOLFOX6 as first-line treatment for patients with advanced colorectal cancer: a double-blind, randomized phase III study (HORIZON III). J Clin Oncol 2012;30:3588-3595.
25. Van Cutsem E, Tabernero J, Lakomy R, et al. Addition of aflibercept to fluorouracil, leucovorin, and irinotecan improves survival in a phase III randomized trial in patients with metastatic colorectal cancer previously treated with an oxaliplatin-based regimen. J Clin Oncol 2012;30: 3499-3506.

26. Saltz LB, Clarke S, Díaz-Rubio $\mathrm{E}$, et al. Bevacizumab in combination with oxaliplatin-based chemotherapy as first-line therapy in metastatic colorectal cancer: a randomized phase III study. J Clin Oncol 2008;26: 2013-2019.

27. Tabernero J, Yoshino T, Cohn AL, et al. Ramucirumab versus placebo in combination with second-line FOLFIRI in patients with metastatic colorectal carcinoma that progressed during or after first-line therapy with bevacizumab, oxaliplatin, and a fluoropyrimidine (RAISE): a randomised, double-blind, multicentre, phase 3 study. Lancet Oncol 2015;16:499-508.

28. Altundag K. Coadministration of proton pump inhibitors and the efficacy of capecitabine in patients with advanced adenocarcinoma of the small bowel or ampulla of vater [letter]. Cancer 2017;123:1074.

29. Wang $X$, Liu C, Wang J, et al. Proton pump inhibitors increase the chemosensitivity of patients with advanced colorectal cancer. Oncotarget 2017;8:58801-58808.

30. Duncan JR, Penman ID, Plumb J, et al. Omeprazole is not cytotoxic to colorectal cancer cells in vitro [abstract]. Gastroenterology 2000;118: Abstract A1386.

31. Nies AT, Hofmann U, Resch $C$, et al. Proton pump inhibitors inhibit metformin uptake by organic cation transporters (OCTs). PLoS One 2011; 6:e22163

32. Han X, Quinney SK, Wang Z, et al. Identification and mechanistic investigation of drug-drug interactions associated with myopathy: a translational approach. Clin Pharmacol Ther 2015;98:321-327.

33. Redzic ZB, Hasan FA, Al-Sarraf H. Effects of omeprazole treatment on nucleoside transporter expression and adenosine uptake in rat gastric mucosa. Can J Physiol Pharmacol 2009;87:402-410.

34. Phua LC, Mal M, Koh PK, et al. Investigating the role of nucleoside transporters in the resistance of colorectal cancer to 5-fluorouracil therapy. Cancer Chemother Pharmacol 2013;71:817-823.

35. Kobayashi K, Bouscarel B, Matsuzaki Y, et al. pH-dependent uptake of irinotecan and its active metabolite, SN-38, by intestinal cells. Int J Cancer 1999;83:491-496

36. van der Bol JM, Loos WJ, de Jong FA, et al. Effect of omeprazole on the pharmacokinetics and toxicities of irinotecan in cancer patients: a prospective cross-over drug-drug interaction study. Eur J Cancer 2011;47: 831-838.

37. Imhann F, Bonder MJ, Vich Vila A, et al. Proton pump inhibitors affect the gut microbiome. Gut 2016;65:740-748.

38. Biswas S, Benedict SH, Lynch SG, et al. Potential immunological consequences of pharmacological suppression of gastric acid production in patients with multiple sclerosis. BMC Med 2012;10:57.

39. Macke L, Schulz C, Koletzko L, et al. Systematic review: the effects of proton pump inhibitors on the microbiome of the digestive tract-evidence from next-generation sequencing studies. Aliment Pharmacol Ther 2020 51:505-526.

40. Wong SH, Yu J. Gut microbiota in colorectal cancer: mechanisms of action and clinical applications. Nat Rev Gastroenterol Hepatol 2019;16: 690-704.

41. Haller DG, Cassidy J, Clarke SJ, et al. Potential regional differences for the tolerability profiles of fluoropyrimidines. J Clin Oncol 2008;26: 2118-2123 

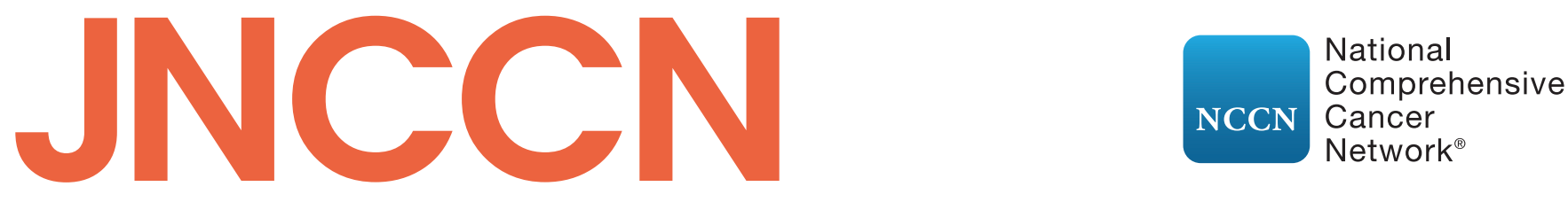

JOURNAL OF THE NATIONAL COMPREHENSIVE CANCER NETWORK

Supplemental online content for:

\section{Proton Pump Inhibitors and Survival in Patients With Colorectal Cancer Receiving Fluoropyrimidine-Based Chemotherapy}

Ganessan Kichenadasse, MBBS, FRACP; John O. Miners, PhD; Arduino A. Mangoni, MBBS, PhD, FRACP;

Christos S. Karapetis, MBBS, FRACP; Ashley M. Hopkins, PhD; and Michael J. Sorich, PhD

J Natl Compr Canc Netw 2021;19(9):1037-1044

eFigure 1: Pooled OS Using Unadjusted Hazard Ratios

eFigure 2: Pooled PFS Using Unadjusted Hazard Ratios

eFigure 3: Subgroup Analysis for OS Using Adjusted Hazard Ratios

eFigure 4: Pooled Response Rates and Concomitant PPI Use

eFigure 5: Pooled Adjusted OS and Concomitant H2RA Use

eFigure 6: Pooled Adjusted PFS and Concomitant H2RA Use

eFigure 7: Pooled Adjusted OR for Response Rates and Concomitant H2RA Use

eTable 1: Included Trials of PPIs and Fluoropyrimidines

eTable 2: Proton Pump Inhibitor Use

eTable 3: Proton Pump Inhibitor Use and Outcomes

eTable 4: H2RA Use and Outcomes

eTable 5: PPI Use and Survival Outcomes in the RAISE Trial With Contemporary Prognostic Factors 


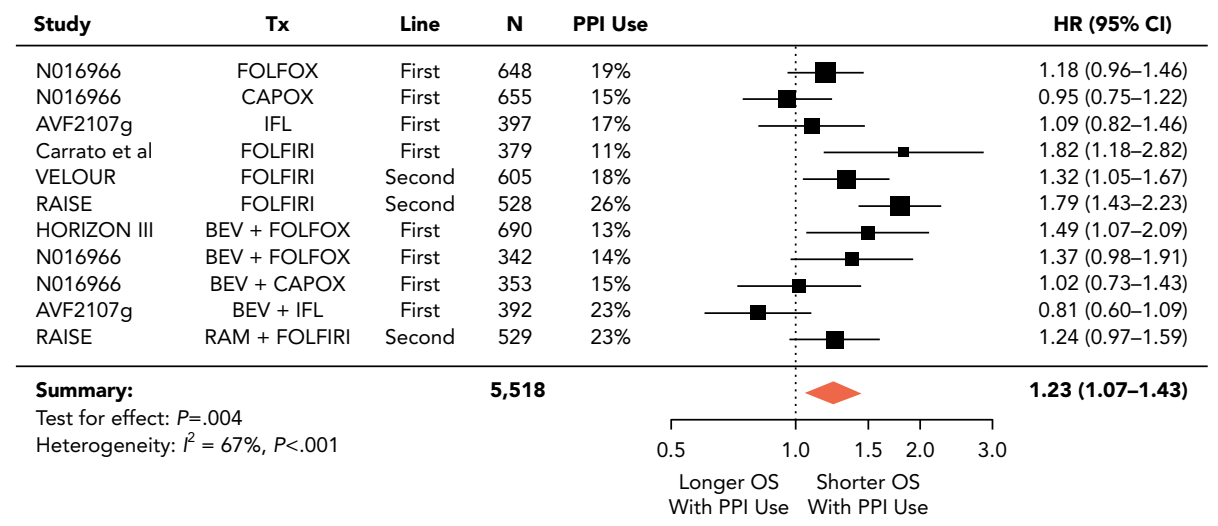

eFigure 1. Pooled OS using unadjusted HRs.

Abbreviations: BEV, bevacizumab; CAPOX, capecitabine/oxaliplatin; FOLFIRI, 5-FU/folinic acid/irinotecan; FOLFOX, 5-FU/leucovorin/oxaliplatin; HR, hazard ratio; $\mathrm{IFL}$, irinotecan/leucovorin/5-FU; OS, overall survival; PPI, proton pump inhibitor; RAM, ramucirumab; Tx, treatment.

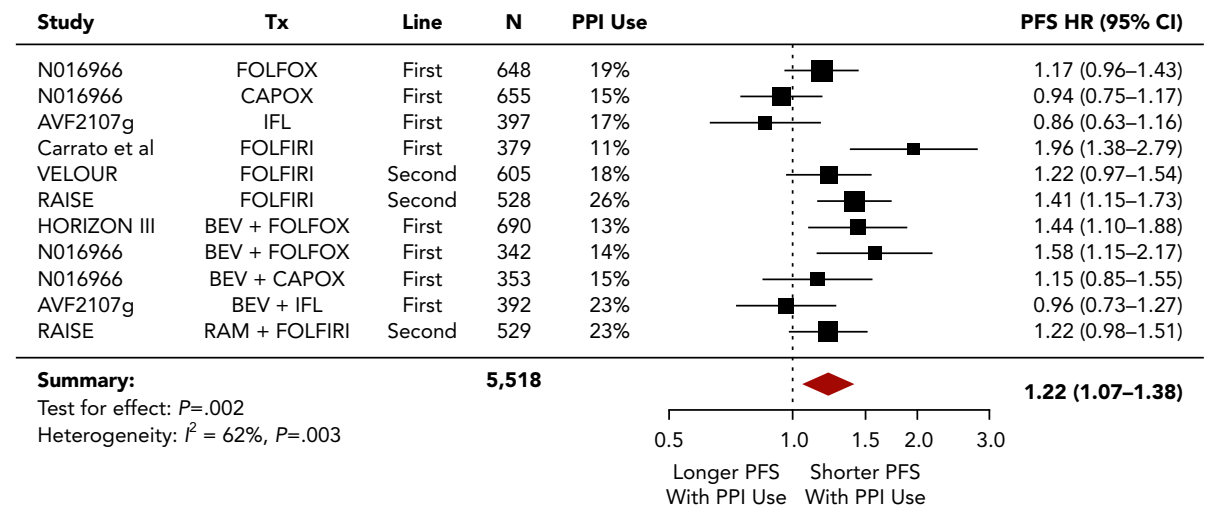

eFigure 2. Pooled PFS using unadjusted HRs.

Abbreviations: BEV, bevacizumab; CAPOX, capecitabine/oxaliplatin; FOLFIRI, 5-FU/folinic acid/irinotecan; FOLFOX, 5-FU/leucovorin/oxaliplatin; HR, hazard ratio; IFL, irinotecan/leucovorin/5-FU; PFS, progression-free survival; PPI, proton pump inhibitor; RAM, ramucirumab; Tx, treatment. 
A

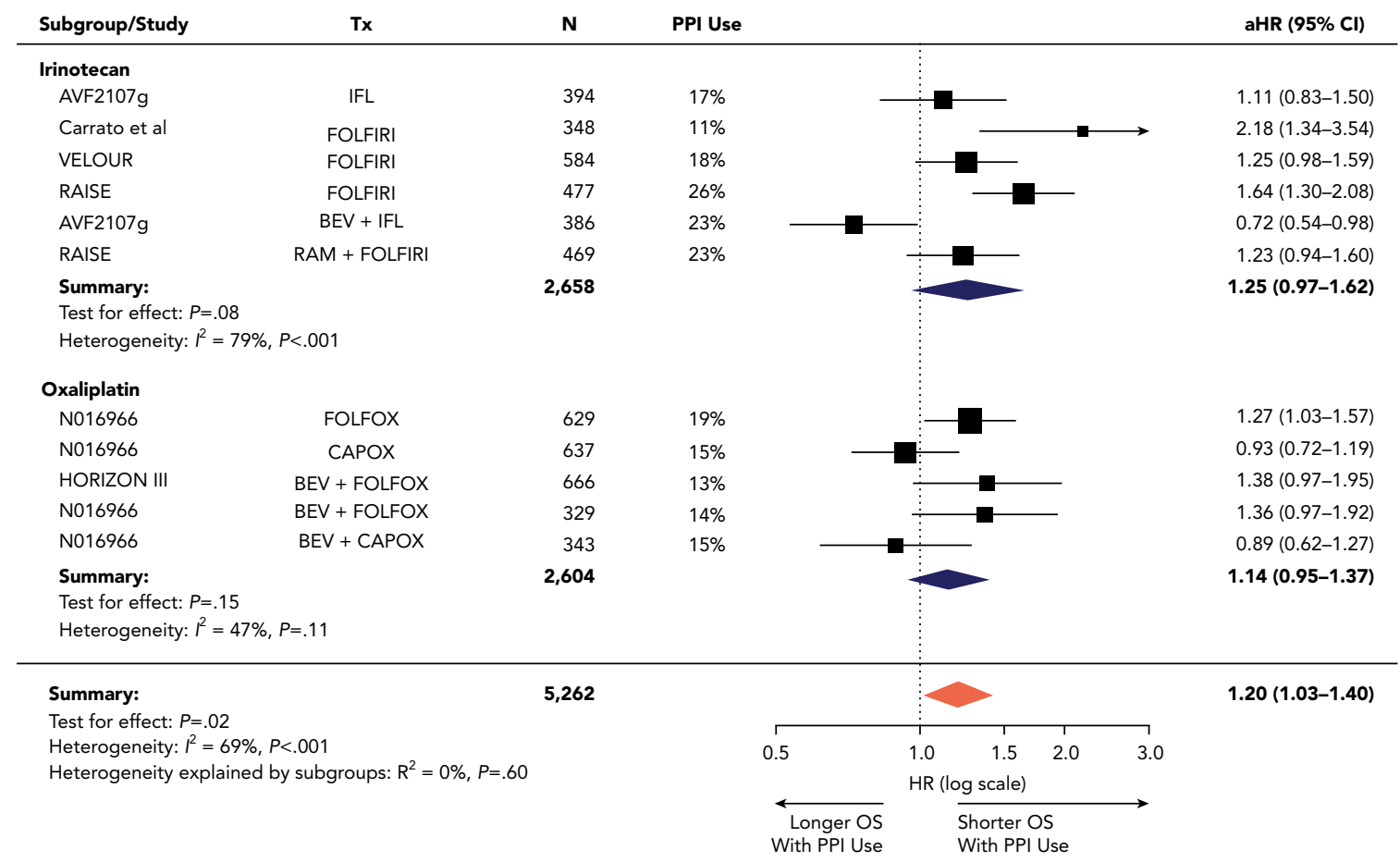

B

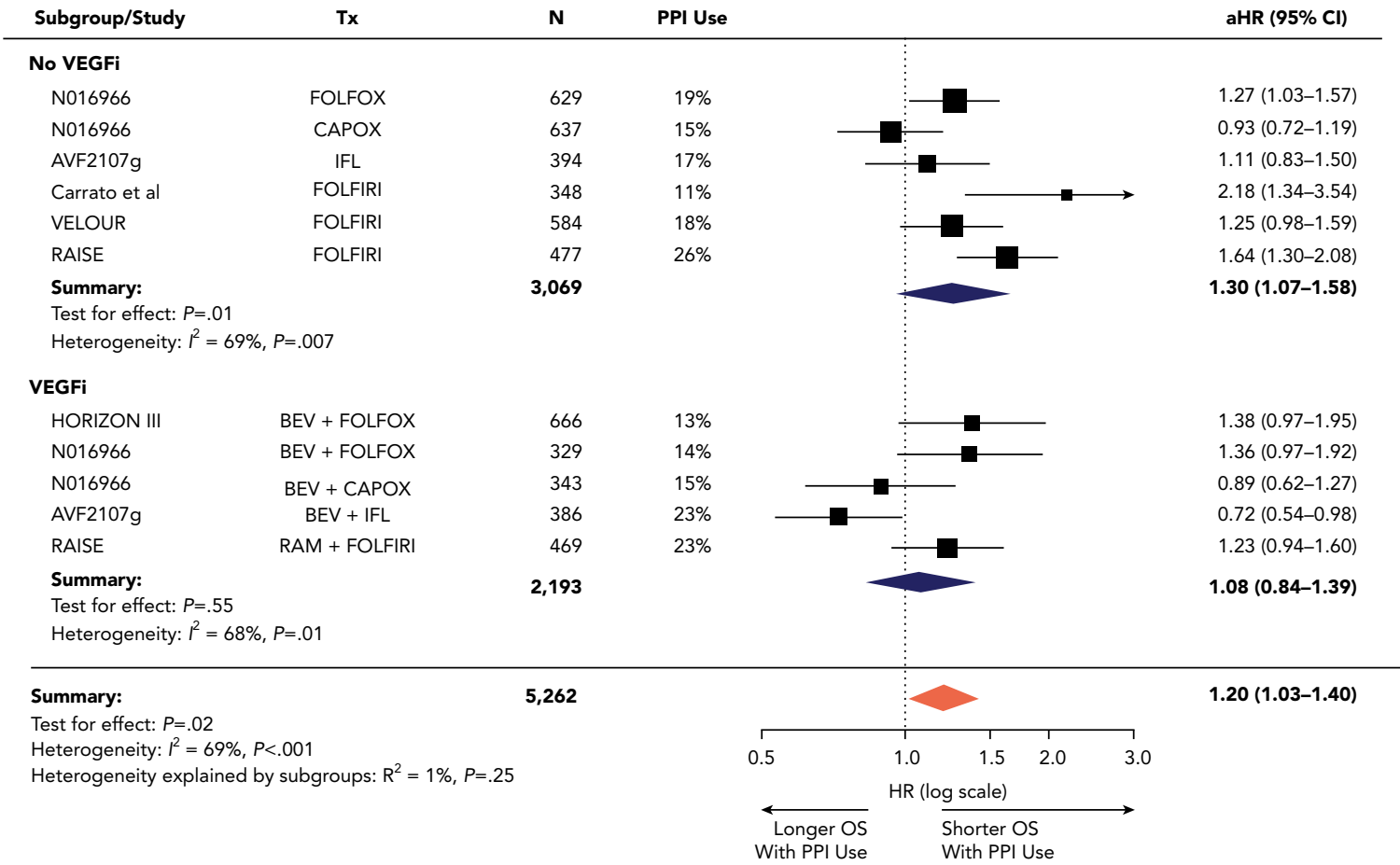

eFigure 3. Subgroup analysis of OS using aHRs: (A) concomitant chemotherapy, (B) concomitant VEGFRi use, (C) by line of use, and (D) capecitabine vs 5 -FU.

Abbreviations: aHR, adjusted hazard ratio; BEV, bevacizumab; CAPOX, capecitabine/oxaliplatin; FOLFIRI, 5-FU/folinic acid/irinotecan; FOLFOX, 5-FU/leucovorin/ oxaliplatin; HR, hazard ratio; IFL, irinotecan/leucovorin/5-FU; OS, overall survival; PPI, proton pump inhibitor; RAM, ramucirumab; Tx, treatment; VEGFRi, vascular endothelial growth factor receptor inhibitor.

(continued on next page) 
C

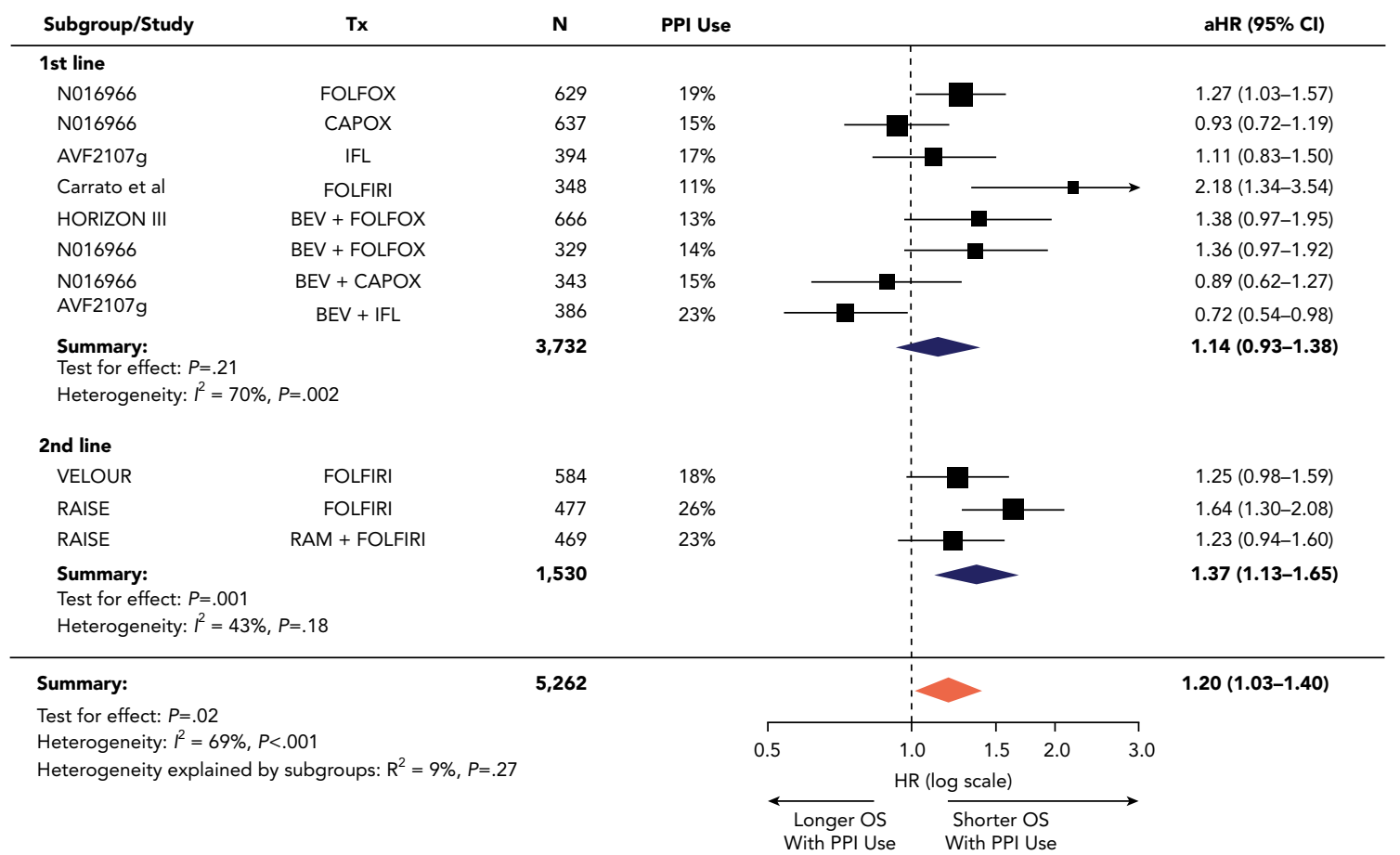

D

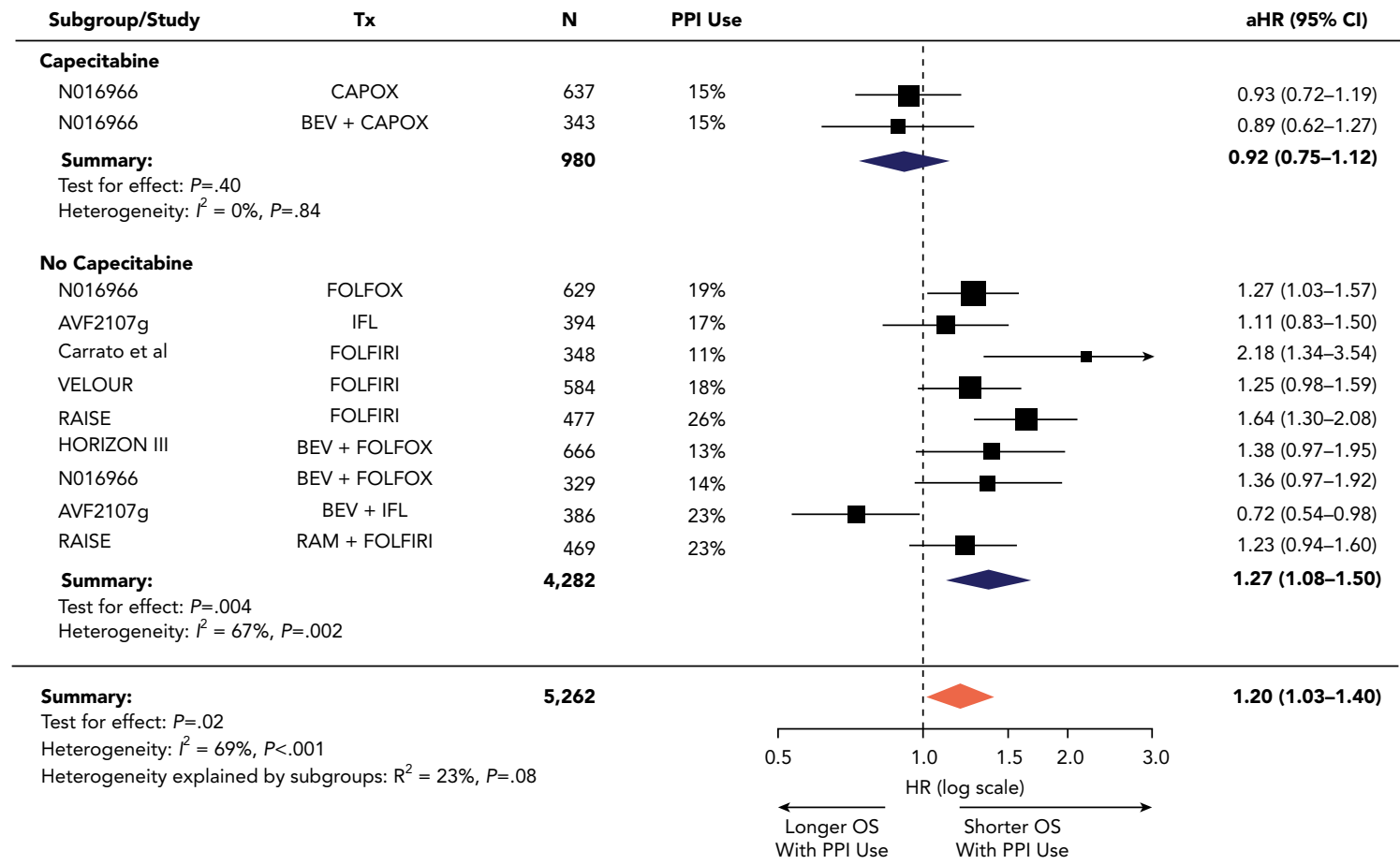

eFigure 3 (continued). Subgroup analysis of OS using aHRs: (A) concomitant chemotherapy, (B) concomitant VEGFRi use, (C) by line of use, and (D) capecitabine vs 5-FU.

Abbreviations: aHR, adjusted hazard ratio; BEV, bevacizumab; CAPOX, capecitabine/oxaliplatin; FOLFIRI, 5-FU/folinic acid/irinotecan; FOLFOX, 5-FU/leucovorin/ oxaliplatin; HR, hazard ratio; IFL, irinotecan/leucovorin/5-FU; OS, overall survival; PPI, proton pump inhibitor; RAM, ramucirumab; Tx, treatment; VEGFRi, vascular endothelial growth factor receptor inhibitor. 


\section{4 - Kichenadasse et al}

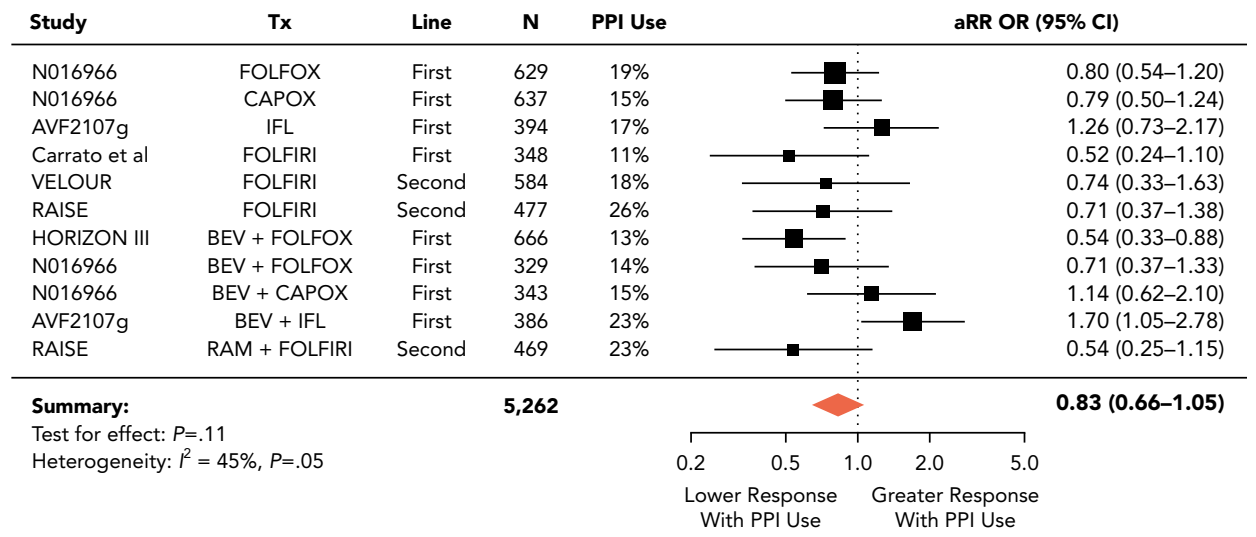

eFigure 4. Pooled response rates and concomitant PPI use.

Abbreviations: aRR, adjusted response rate; BEV, bevacizumab; CAPOX, capecitabine/oxaliplatin; FOLFIRI, 5-FU/folinic acid/irinotecan; FOLFOX, 5-FU/leucovorin/ oxaliplatin; IFL, irinotecan/leucovorin/5-FU; OR, odds ratio; PPI, proton pump inhibitor; RAM, ramucirumab; Tx, treatment.

\begin{tabular}{|c|c|c|c|c|c|c|c|}
\hline Study & Tx & Line & $\mathbf{N}$ & H2RA \% & HR $(95 \% \mathrm{Cl})$ & No H2RA & H2RA use \\
\hline N016966 & FOLFOX & First & 629 & $5.7 \%$ & $1.19(0.83-1.71)$ & & - \\
\hline N016966 & CAPOX & First & 637 & $6 \%$ & $0.83(0.59-1.16)$ & & \\
\hline AVF2107g & $\mathrm{IFL}$ & First & 394 & $17 \%$ & $0.87(0.64-1.18)$ & & \\
\hline Carrato et al & FOLFIRI & First & 348 & $4.2 \%$ & $1.39(0.56-3.46)$ & & \\
\hline VELOUR & FOLFIRI & Second & 584 & $2.8 \%$ & $1.02(0.60-1.74)$ & & \\
\hline RAISE & FOLFIRI & Second & 469 & $4.5 \%$ & $1.42(0.85-2.38)$ & & \\
\hline HORIZON III & BEV + FOLFOX & First & 666 & $2.2 \%$ & $1.06(0.43-2.62)$ & & \\
\hline N016966 & BEV + FOLFOX & First & 329 & $5.6 \%$ & $0.99(0.60-1.64)$ & & \\
\hline N016966 & BEV + CAPOX & First & 343 & $6.8 \%$ & $1.07(0.70-1.64)$ & & \\
\hline AVF2107g & $\mathrm{BEV}+\mathrm{IFL}$ & First & 386 & $16 \%$ & $0.86(0.62-1.20)$ & & \\
\hline RAISE & RAM + FOLFIRI & Second & 477 & $3.2 \%$ & $0.37(0.05-2.87)$ & & \\
\hline Total (fixed ef & & & 5,262 & & $0.98(0.86-1.12)$ & & \\
\hline Total (random & & & & & $0.98(0.86-1.12)$ & & \\
\hline \multirow{2}{*}{\multicolumn{6}{|c|}{ Heterogeneity: $\chi_{10}^{2}=6.84(P=.74), I^{2}=0 \%$}} & 0.5 & $\begin{array}{lll} & 1 & 1 \\
0 & 1.5 & 3.0\end{array}$ \\
\hline & & & & & & & $\% \mathrm{Cl})$ \\
\hline
\end{tabular}

eFigure 5. Pooled adjusted OS and concomitant H2RA use.

Abbreviation: BEV, bevacizumab; CAPOX, capecitabine/oxaliplatin; FOLFIRI, 5-FU/folinic acid/irinotecan; FOLFOX, 5-FU/leucovorin/oxaliplatin; H2RA, histamine $\mathrm{H}$ 2-receptor antagonists; HR, hazard ratio; IFL, irinotecan/leucovorin/5-FU; RAM, ramucirumab; Tx, treatment. 


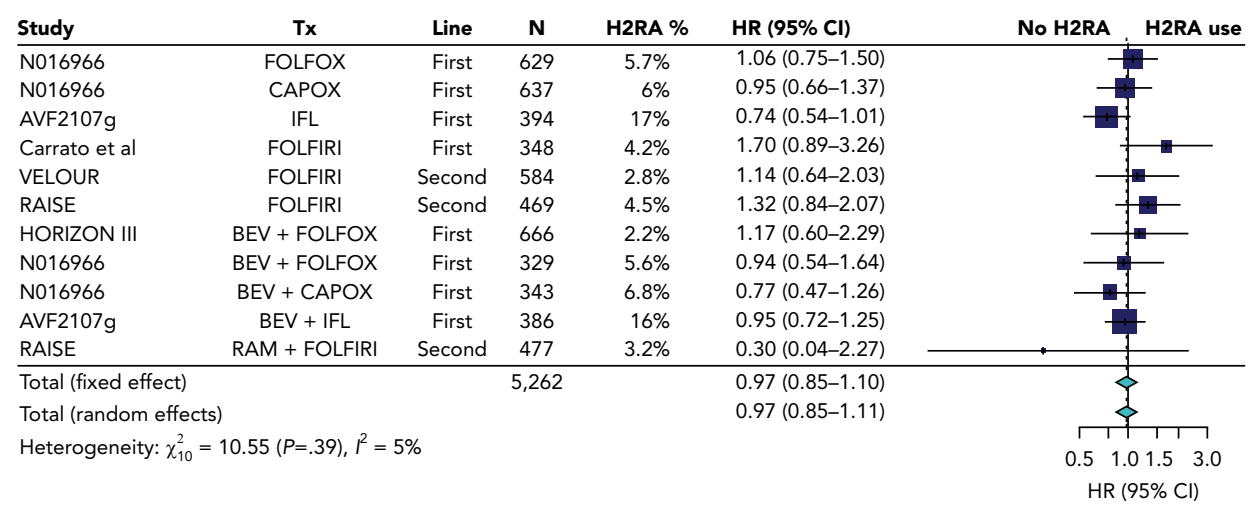

eFigure 6. Pooled adjusted PFS and concomitant H2RA use.

Abbreviation: BEV, bevacizumab; CAPOX, capecitabine/oxaliplatin; FOLFIRI, 5-FU/folinic acid/irinotecan; FOLFOX, 5-FU/leucovorin/oxaliplatin; H2RA, histamine $\mathrm{H} 2$-receptor antagonists; HR, hazard ratio; IFL, irinotecan/leucovorin/5-FU; PFS, progression-free survival; RAM, ramucirumab; Tx, treatment.

\begin{tabular}{|c|c|c|c|c|c|c|c|}
\hline Study & $\mathbf{T x}$ & Line & $\mathbf{N}$ & H2RA \% & $\mathrm{HR}(95 \% \mathrm{Cl})$ & No H2RA & H2RA use \\
\hline N016966 & FOLFOX & First & 629 & $5.7 \%$ & $1.48(0.73-2.99)$ & & - \\
\hline N016966 & CAPOX & First & 637 & $6 \%$ & $1.53(0.79-2.97)$ & & \\
\hline AVF2107g & IFL & First & 394 & $17 \%$ & $1.45(0.84-2.50)$ & & \\
\hline Carrato et al & FOLFIRI & First & 348 & $4.2 \%$ & $0.55(0.16-1.91)$ & 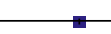 & \\
\hline VELOUR & FOLFIRI & Second & 584 & $2.8 \%$ & $0.62(0.08-4.88)$ & & \\
\hline RAISE & FOLFIRI & Second & 469 & $4.5 \%$ & $0.30(0.04-2.27)$ & & \\
\hline HORIZON III & BEV + FOLFOX & First & 666 & $2.2 \%$ & $0.52(0.16-1.69)$ & \#- & \\
\hline N016966 & BEV + FOLFOX & First & 329 & $5.6 \%$ & $0.81(0.30-2.17)$ & & \\
\hline N016966 & BEV + CAPOX & First & 343 & $6.8 \%$ & $0.92(0.39-2.17)$ & & \\
\hline AVF2107g & $\mathrm{BEV}+\mathrm{IFL}$ & First & 386 & $16 \%$ & $1.52(0.87-2.66)$ & & \\
\hline RAISE & RAM + FOLFIRI & Second & 477 & $3.2 \%$ & $0.37(0.05-2.81)$ & & \\
\hline Total (fixed ef & & & 5,262 & & $1.18(0.91-1.52)$ & & $>$ \\
\hline Total (random & & & & & $1.18(0.91-1.52)$ & & $\dot{\theta}$ \\
\hline \multirow{2}{*}{\multicolumn{6}{|c|}{ Heterogeneity: $\chi_{10}^{2}=9.90(P=.45), I^{2}=0 \%$}} & 0.5 & 01.53 .0 \\
\hline & & & & & & \multicolumn{2}{|c|}{ OR $(95 \% \mathrm{Cl})$} \\
\hline
\end{tabular}

eFigure 7. Pooled adjusted OR for response rates and concomitant H2RA use.

Abbreviation: BEV, bevacizumab; CAPOX, capecitabine/oxaliplatin; FOLFIRI, 5-FU/folinic acid/irinotecan; FOLFOX, 5-FU/leucovorin/oxaliplatin; H2RA, histamine H2-receptor antagonists; HR, hazard ratio; IFL, irinotecan/leucovorin/5-FU; OR, odds ratio; RAM, ramucirumab; Tx, treatment. 
eTable 1. Included Trials of PPls and Fluoropyrimidines

\begin{tabular}{|c|c|c|c|c|c|}
\hline Trial & Population & Treatment Intervention & $\begin{array}{c}\text { Cohort Size } \\
n\end{array}$ & $\begin{array}{l}\text { PPI Use } \\
\text { n (\%) }\end{array}$ & Comments \\
\hline \multicolumn{6}{|l|}{ First-line trials } \\
\hline N016966 2 & Advanced CRC, treatment-naïve & $\begin{array}{l}\text { FOLFOX-4 } \pm \text { placebo } \\
\text { CAPOX } \pm \text { placebo } \\
\text { FOLFOX-4 + BEV } \\
\text { CAPOX + BEV }\end{array}$ & $\begin{array}{c}668 \\
667 \\
350 \\
350\end{array}$ & $\begin{array}{r}123(18.4) \\
99(14.8) \\
47(13.4) \\
53(15.1)\end{array}$ & All arms included \\
\hline HORIZON III & Advanced CRC, treatment-naïve & FOLFOX-4 + BEV & 713 & $88(12.3)$ & Control arm only \\
\hline \multicolumn{6}{|l|}{ Second-line trials } \\
\hline VELOUR 5 & Prior oxaliplatin chemotherapy & FOLFIRI & 614 & $113(18.4)$ & Control arm only \\
\hline RAISE $^{6}$ & Prior oxaliplatin chemotherapy & $\begin{array}{l}\text { FOLFIRI + placebo } \\
\text { FOLFIRI + RAM }\end{array}$ & $\begin{array}{l}536 \\
536\end{array}$ & $\begin{array}{l}136(25.3) \\
122(22.7)\end{array}$ & All arms included \\
\hline
\end{tabular}

Abbreviations: BEV, bevacizumab; CAPOX, capecitabine/oxaliplatin; CRC, colorectal cancer; FOLFIRI, 5-FU/folinic acid/irinotecan; FOLFOX, 5-FU/leucovorin/ oxaliplatin; IFL, irinotecan/leucovorin/5-FU; PPI, proton pump inhibitor; RAM, ramucirumab.

\section{References}

1. Hurwitz H, Fehrenbacher L, Novotny W, et al. Bevacizumab plus irinotecan, fluorouracil, and leucovorin for metastatic colorectal cancer. N Engl J Med 2004;350: 2335-2342.

2. Saltz LB, Clarke S, Díaz-Rubio E, et al. Bevacizumab in combination with oxaliplatin-based chemotherapy as first-line therapy in metastatic colorectal cancer: a randomized phase III study. J Clin Oncol 2008;26:2013-2019.

3. Carrato A, Swieboda-Sadlej A, Staszewska-Skurczynska M, et al. Fluorouracil, leucovorin, and irinotecan plus either sunitinib or placebo in metastatic colorectal cancer: a randomized, phase III trial. J Clin Oncol 2013;31:1341-1347.

4. Schmoll HJ, Cunningham D, Sobrero A, et al. Cediranib with mFOLFOX6 versus bevacizumab with mFOLFOX6 as first-line treatment for patients with advanced colorectal cancer: a double-blind, randomized phase III study (HORIZON III). J Clin Oncol 2012;30:3588-3595.

5. Van Cutsem E, Tabernero J, Lakomy R, et al. Addition of aflibercept to fluorouracil, leucovorin, and irinotecan improves survival in a phase III randomized trial in patients with metastatic colorectal cancer previously treated with an oxaliplatin-based regimen. $\mathrm{J}$ Clin Oncol 2012;30:3499-3506.

6. Tabernero J, Yoshino T, Cohn AL, et al. Ramucirumab versus placebo in combination with second-line FOLFIRl in patients with metastatic colorectal carcinoma that progressed during or after first-line therapy with bevacizumab, oxaliplatin, and a fluoropyrimidine (RAISE): a randomised, double-blind, multicentre, phase 3 study. Lancet Oncol 2015;16:499-508. 


\section{eTable 2. Proton Pump Inhibitor Use}

\begin{tabular}{|c|c|c|c|c|c|c|}
\hline $\begin{array}{l}\text { Proton Pump } \\
\text { Inhibitor }\end{array}$ & $\begin{array}{c}\text { AVF2107g } \\
n(\%)\end{array}$ & $\begin{array}{c}\text { N016966 } \\
\text { n (\%) }\end{array}$ & $\begin{array}{c}\text { Carrato et } \mathrm{al}^{3} \\
\mathrm{n}(\%)\end{array}$ & $\begin{array}{c}\text { HORIZON III } \\
\text { n (\%) }\end{array}$ & $\begin{array}{l}\text { VELOUR }^{5} \\
\text { n (\%) }\end{array}$ & $\begin{array}{l}\text { RAISE6 }^{6} \\
\text { n (\%) }\end{array}$ \\
\hline Total, N & 159 & 327 & 43 & 88 & 113 & 258 \\
\hline Esomeprazole & $29(18.2)$ & $34(10.3)$ & $6(13.9)$ & $10(11.4)$ & $13(11.5)$ & $18(6.9)$ \\
\hline Lansoprazole & $60(37.7)$ & $48(14.6)$ & $3(6.9)$ & 15 (17.0) & $17(15.0)$ & $47(18.2)$ \\
\hline Omeprazole & $46(28.9)$ & $115(35.1)$ & 22 (51.1) & $36(40.9)$ & $59(52.2)$ & 119 (46.1) \\
\hline Pantoprazole & $31(19.4)$ & 38 (11.6) & $12(2.9)$ & $22(24.9)$ & $21(18.5)$ & $56(21.7)$ \\
\hline Rabeprazole & $10(6.2)$ & $8(2.4)$ & $0(0)$ & $5(5.7)$ & $3(2.6)$ & $18(6.9)$ \\
\hline
\end{tabular}

\section{References}

1. Hurwitz H, Fehrenbacher L, Novotny W, et al. Bevacizumab plus irinotecan, fluorouracil, and leucovorin for metastatic colorectal cancer. N Engl J Med 2004;350: 2335-2342.

2. Saltz LB, Clarke S, Díaz-Rubio E, et al. Bevacizumab in combination with oxaliplatin-based chemotherapy as first-line therapy in metastatic colorectal cancer: a randomized phase III study. J Clin Oncol 2008;26:2013-2019.

3. Carrato A, Swieboda-Sadlej A, Staszewska-Skurczynska M, et al. Fluorouracil, leucovorin, and irinotecan plus either sunitinib or placebo in metastatic colorectal cancer: a randomized, phase III trial. J Clin Oncol 2013;31:1341-1347.

4. Schmoll HJ, Cunningham D, Sobrero A, et al. Cediranib with mFOLFOX6 versus bevacizumab with mFOLFOX6 as first-line treatment for patients with advanced colorectal cancer: a double-blind, randomized phase III study (HORIZON III). J Clin Oncol 2012;30:3588-3595.

5. Van Cutsem E, Tabernero J, Lakomy R, et al. Addition of aflibercept to fluorouracil, leucovorin, and irinotecan improves survival in a phase III randomized trial in patients with metastatic colorectal cancer previously treated with an oxaliplatin-based regimen. J Clin Oncol 2012;30:3499-3506.

6. Tabernero J, Yoshino T, Cohn AL, et al. Ramucirumab versus placebo in combination with second-line FOLFIRI in patients with metastatic colorectal carcinoma that progressed during or after first-line therapy with bevacizumab, oxaliplatin, and a fluoropyrimidine (RAISE): a randomised, double-blind, multicentre, phase 3 study. Lancet Oncol 2015;16:499-508. 
eTable 3. Proton Pump Inhibitor Use and Outcomes

\begin{tabular}{|c|c|c|c|}
\hline Trial & $\begin{array}{l}\text { Unadjusted OS } \\
\text { HR }(95 \% \mathrm{Cl})\end{array}$ & $\begin{array}{c}\text { Unadjusted PFS } \\
\text { HR }(95 \% \mathrm{Cl})\end{array}$ & $\begin{array}{c}\text { Unadjusted Objective RR } \\
\text { OR }(95 \% \mathrm{Cl})\end{array}$ \\
\hline \multicolumn{4}{|l|}{ AVF2107g ${ }^{1}$} \\
\hline IFL & $1.09(0.82-1.46)$ & $0.86(0.63-1.16)$ & $1.26(0.74-2.15)$ \\
\hline $\mathrm{IFL}+\mathrm{BEV}$ & $0.81(0.60-1.09)$ & $0.96(0.73-1.27)$ & $1.59(0.98-2.55)$ \\
\hline \multicolumn{4}{|l|}{ N016966² } \\
\hline CAPOX & $0.95(0.75-1.22)$ & $0.94(0.75-1.17)$ & $0.80(0.52-1.23)$ \\
\hline FOLFOX & $1.18(0.96-1.46)$ & $1.17(0.96-1.43)$ & $0.79(0.54-1.18)$ \\
\hline CAPOX + BEV & $1.02(0.73-1.43)$ & $1.15(0.85-1.55)$ & $1.01(0.56-1.81)$ \\
\hline FOLFOX + BEV & $1.37(0.98-1.91)$ & $1.58(1.15-2.17)$ & $0.73(0.39-1.35)$ \\
\hline \multicolumn{4}{|l|}{ Carrato et $\mathrm{al}^{3}$} \\
\hline FOLFIRI & $1.82(1.18-2.82)$ & $1.96(1.38-2.78)$ & $0.52(0.25-1.06)$ \\
\hline \multicolumn{4}{|l|}{ VELOUR ${ }^{4}$} \\
\hline FOLFIRI & $1.32(1.05-1.67)$ & $1.22(0.97-1.54)$ & $0.66(0.30-1.45)$ \\
\hline \multicolumn{4}{|l|}{ RAISE $^{5}$} \\
\hline FOLFIRI & $1.79(1.43-2.23)$ & $1.41(1.15-1.72)$ & $0.66(0.35-1.25)$ \\
\hline FOLFIRI + RAM & $1.24(0.97-1.59)$ & $1.22(0.98-1.51)$ & $0.56(0.28-1.11)$ \\
\hline \multicolumn{4}{|l|}{ HORIZON III' } \\
\hline FOLFOX + BEV & $1.49(1.07-2.09)$ & $1.44(1.10-1.88)$ & $0.51(0.32-0.82)$ \\
\hline
\end{tabular}

Abbreviations: BEV, bevacizumab; CAPOX, capecitabine/oxaliplatin; FOLFIRI, 5-FU/folinic acid/irinotecan; FOLFOX, 5-FU/leucovorin/oxaliplatin; HR, hazard ratio; $\mathrm{IFL}$, irinotecan/leucovorin/5-FU; OS, overall survival; PFS, progression-free survival; RAM, ramucirumab; RR, response rate.

\section{References}

1. Hurwitz H, Fehrenbacher L, Novotny W, et al. Bevacizumab plus irinotecan, fluorouracil, and leucovorin for metastatic colorectal cancer. N Engl J Med 2004;350: 2335-2342.

2. Saltz LB, Clarke S, Díaz-Rubio E, et al. Bevacizumab in combination with oxaliplatin-based chemotherapy as first-line therapy in metastatic colorectal cancer: a randomized phase III study. J Clin Oncol 2008;26:2013-2019.

3. Carrato A, Swieboda-Sadlej A, Staszewska-Skurczynska M, et al. Fluorouracil, leucovorin, and irinotecan plus either sunitinib or placebo in metastatic colorectal cancer: a randomized, phase III trial. J Clin Oncol 2013;31:1341-1347.

4. Van Cutsem E, Tabernero J, Lakomy R, et al. Addition of aflibercept to fluorouracil, leucovorin, and irinotecan improves survival in a phase III randomized trial in patients with metastatic colorectal cancer previously treated with an oxaliplatin-based regimen. J Clin Oncol 2012;30:3499-3506.

5. Tabernero J, Yoshino T, Cohn AL, et al. Ramucirumab versus placebo in combination with second-line FOLFIRI in patients with metastatic colorectal carcinoma that progressed during or after first-line therapy with bevacizumab, oxaliplatin, and a fluoropyrimidine (RAISE): a randomised, double-blind, multicentre, phase 3 study. Lancet Oncol 2015;16:499-508.

4. Schmoll HJ, Cunningham D, Sobrero A, et al. Cediranib with mFOLFOX6 versus bevacizumab with mFOLFOX6 as first-line treatment for patients with advanced colorectal cancer: a double-blind, randomized phase III study (HORIZON III). J Clin Oncol 2012;30:3588-3595. 


\section{eTable 4. H2RA Use and Outcomes}

\begin{tabular}{|c|c|c|c|}
\hline Trial & $\begin{array}{l}\text { Adjusted OS } \\
\mathrm{HR}(95 \% \mathrm{Cl})\end{array}$ & $\begin{array}{l}\text { Adjusted PFS } \\
\text { HR }(95 \% \mathrm{Cl})\end{array}$ & $\begin{array}{c}\text { Adjusted Objective RR } \\
\text { OR }(95 \% \mathrm{Cl})\end{array}$ \\
\hline \multicolumn{4}{|c|}{ AVF2107g $(n=129 ; 5.9 \%)$} \\
\hline IFL & $0.87(0.64-1.18)$ & $0.74(0.55-1.02)$ & $1.45(0.84-2.50)$ \\
\hline $\mathrm{IFL}+\mathrm{BEV}$ & $0.86(0.62-1.20)$ & $0.81(0.59-1.12)$ & $1.53(0.87-2.67)$ \\
\hline \multicolumn{4}{|c|}{$N 016966^{2}(n=120 ; 5.9 \%)$} \\
\hline CAPOX & $0.95(0.66-1.38)$ & $0.83(0.59-1.16)$ & $1.53(0.79-2.98)$ \\
\hline FOLFOX & $1.19(0.83-1.71)$ & $1.19(0.86-1.66)$ & $1.48(0.73-2.97)$ \\
\hline CAPOX + BEV & $0.77(0.47-1.26)$ & $1.07(0.70-1.65)$ & $0.92(0.39-2.16)$ \\
\hline FOLFOX + BEV & $0.94(0.54-1.64)$ & $0.99(0.60-1.65)$ & $0.81(0.30-2.16)$ \\
\hline \multicolumn{4}{|c|}{ Carrato et $\mathrm{al}^{3}(n=16 ; 4.2 \%)$} \\
\hline FOLFIRI & $1.39(0.56-3.48)$ & $1.70(0.88-3.27)$ & $0.56(0.16-1.92)$ \\
\hline \multicolumn{4}{|c|}{ VELOUR $^{4}(n=17 ; 2.8 \%)$} \\
\hline FOLFIRI & $1.02(0.59-1.75)$ & $1.14(0.64-2.03)$ & $0.62(0.08-4.95)$ \\
\hline \multicolumn{4}{|l|}{ RAISE $^{5}(n=65 ; 6.1 \%)$} \\
\hline FOLFIRI & $0.84(0.47-1.51)$ & $0.91(0.55-1.52)$ & $0.37(0.05-2.88)$ \\
\hline FOLFIRI + RAM & $1.42(0.85-2.38)$ & $1.32(0.84-2.07)$ & $0.30(0.04-2.30)$ \\
\hline \multicolumn{4}{|c|}{ HORIZON III6 ( $n=15 ; 2.2 \%)$} \\
\hline FOLFOX + BEV & $1.06(0.43-2.63)$ & $1.17(0.60-2.29)$ & $0.51(0.16-1.69)$ \\
\hline
\end{tabular}

Abbreviations: BEV, bevacizumab; CAPOX, capecitabine/oxaliplatin; FOLFIRI, 5-FU/folinic acid/irinotecan; FOLFOX, 5-FU/leucovorin/oxaliplatin; H2RA, histamine H2-receptor antagonists; HR, hazard ratio; IFL, irinotecan/leucovorin/5-FU; OS, overall survival; PFS, progression-free survival; RAM, ramucirumab; RR, response rate.

\section{References}

1. Hurwitz H, Fehrenbacher L, Novotny W, et al. Bevacizumab plus irinotecan, fluorouracil, and leucovorin for metastatic colorectal cancer. N Engl J Med 2004;350: 2335-2342.

2. Saltz LB, Clarke S, Díaz-Rubio E, et al. Bevacizumab in combination with oxaliplatin-based chemotherapy as first-line therapy in metastatic colorectal cancer: a randomized phase III study. J Clin Oncol 2008;26:2013-2019.

3. Carrato A, Swieboda-Sadlej A, Staszewska-Skurczynska M, et al. Fluorouracil, leucovorin, and irinotecan plus either sunitinib or placebo in metastatic colorectal cancer: a randomized, phase III trial. J Clin Oncol 2013;31:1341-1347.

4. Van Cutsem E, Tabernero J, Lakomy R, et al. Addition of aflibercept to fluorouracil, leucovorin, and irinotecan improves survival in a phase III randomized trial in patients with metastatic colorectal cancer previously treated with an oxaliplatin-based regimen. J Clin Oncol 2012;30:3499-3506.

5. Tabernero J, Yoshino T, Cohn AL, et al. Ramucirumab versus placebo in combination with second-line FOLFIRI in patients with metastatic colorectal carcinoma that progressed during or after first-line therapy with bevacizumab, oxaliplatin, and a fluoropyrimidine (RAISE): a randomised, double-blind, multicentre, phase 3 study. Lancet Oncol 2015;16:499-508.

6. Schmoll HJ, Cunningham D, Sobrero A, et al. Cediranib with mFOLFOX6 versus bevacizumab with mFOLFOX6 as first-line treatment for patients with advanced colorectal cancer: a double-blind, randomized phase III study (HORIZON III). J Clin Oncol 2012;30:3588-3595. 


\section{eTable 5. PPI Use and Survival Outcomes in the RAISE Trial ${ }^{1}$ With Contemporary Prognostic Factors}

Adjusted OS

Adjusted PFS

\begin{tabular}{|c|c|c|c|c|}
\hline \multirow{2}{*}{ RAISE Trial Cohorts } & & \\
\hline & $\mathrm{HR}(95 \% \mathrm{Cl})$ & $P$ Value & HR $(95 \%$ Cl) & $P$ Value \\
\hline \multicolumn{5}{|l|}{ FOLFIRI alone } \\
\hline PPI use & $1.63(1.27-2.09)$ & .0001 & 1.35 (1.07-1.69) & .01 \\
\hline Age & $0.99(0.98-1.00)$ & .30 & $0.99(0.80-0.99)$ & .02 \\
\hline Female & $1.07(0.85-1.34)$ & .57 & $1.05(0.85-1.28)$ & .67 \\
\hline White race & $1.30(1.01-1.67)$ & .04 & $0.87(0.70-1.10)$ & .23 \\
\hline ECOG PS & $1.33(1.06-1.67)$ & .01 & $1.18(0.96-1.45)$ & .11 \\
\hline Right-sided primary & $1.07(0.84-1.35)$ & .60 & $1.02(0.83-1.26)$ & .84 \\
\hline Liver metastases & $0.82(0.60-1.13)$ & .23 & $1.11(0.84-1.45)$ & .46 \\
\hline KRAS wild-type & $0.89(0.71-1.11)$ & .29 & $0.97(0.80-1.12)$ & .79 \\
\hline $\mathrm{LDH}>\mathrm{ULN}$ & $1.37(1.07-1.76)$ & .01 & $1.16(0.93-1.46)$ & .18 \\
\hline$A L P>U L N$ & 1.35 (1.06-1.73) & .01 & $1.28(1.02-1.60)$ & .03 \\
\hline$C E A \geq 200 \mu \mathrm{g} / \mathrm{L}$ & $1.52(1.16-2.01)$ & .002 & $1.34(1.03-1.75)$ & .03 \\
\hline \multicolumn{5}{|l|}{ FOLFIRI + RAM } \\
\hline PPI use & $1.16(0.88-1.53)$ & .29 & $1.22(0.96-1.55)$ & .11 \\
\hline Age & $0.99(0.98-1.00)$ & .08 & $0.99(0.98-1.00)$ & .45 \\
\hline Female & $0.79(0.62-1.00)$ & .05 & $0.84(0.68-1.03)$ & .87 \\
\hline White race & $1.25(0.96-1.63)$ & .10 & $0.92(0.73-1.16)$ & .49 \\
\hline ECOG PS & $1.23(0.97-1.50)$ & .08 & $1.17(0.96-1.44)$ & .12 \\
\hline Right-sided primary & $1.40(1.08-1.80)$ & .009 & $1.18(0.94-1.48)$ & .15 \\
\hline Liver metastases & $0.75(0.53-1.03)$ & .08 & $0.91(0.69-1.12)$ & .54 \\
\hline KRAS wild-type & $0.72(0.57-0.91)$ & .006 & $0.87(0.71-1.06)$ & .16 \\
\hline $\mathrm{LDH}>\mathrm{ULN}$ & $2.47(1.90-3.20)$ & $<.0001$ & $1.58(1.27-1.97)$ & $<.0001$ \\
\hline$A L P>U L N$ & $1.55(1.19-2.01)$ & .001 & $1.13(0.89-1.42)$ & .31 \\
\hline$C E A \geq 200 \mu \mathrm{g} / \mathrm{L}$ & $1.43(1.09-1.89)$ & .01 & $1.35(1.05-1.73)$ & .02 \\
\hline
\end{tabular}

Abbreviations: ALP, alkaline phosphatase; FOLFIRI, 5-FU/folinic acid/irinotecan; HR, hazard ratio; LDH, lactate dehydrogenase; OS, overall survival; PFS, progressionfree survival; PPI, proton pump inhibitor; PS, performance status; RAM, ramucirumab; ULN, upper limit of normal.

\section{References}

1. Tabernero J, Yoshino T, Cohn AL, et al. Ramucirumab versus placebo in combination with second-line FOLFIRI in patients with metastatic colorectal carcinoma that progressed during or after first-line therapy with bevacizumab, oxaliplatin, and a fluoropyrimidine (RAISE): a randomised, double-blind, multicentre, phase 3 study. Lancet Oncol 2015;16:499-508. 